\title{
The braid group: redefining
}

\author{
Mahyuddin KM Nasution* \\ Universitas Sumatera Utara, Teknologi Informasi, Fasilkom-TI, Padang Bulan 20155 USU, Medan, Indonesia
}

\begin{abstract}
The role of the braid group constitutes one of the invariant measurements. Through the classification of braids formed several parts of the braid group, but does not computationally distinguish them. Some characteristics have been expressed to give the features to a braid in braid group based on redefining relation between braid group and permutation group.
\end{abstract}

\section{Introduction}

One of technologies that has been growing ever since is the braid $[1,2]$. The braid is widely used in everyday life [3]. The braid is not only used by the public in general, but the academics, especially the biologists for genetic engineering [4]. As a technology, the braid has its own mathematical model [5]. The modelling is to express the privilege of a braid.

In group theory, the braid is one of the most interesting implementations [6], which provides a form of the invariant measurement about a structure [5]. Thus, a braid has the general and special characteristics, which characterizes each braid so as to give the idea of meaning to the structure formed. Therefore, this paper redefines relations between groups based on the braids in the braid groups specifically and expresses the computational characteristics associated with it.

\section{The Review and Motivation}

\subsection{Woven and braid}

Three woven patterns are $\sigma_{i}{ }^{1}, \sigma_{i}{ }^{0}$, and $\sigma_{i}^{-1}$ each like Fig. 1. They build a system if there are two parallel lines $L_{1}$ and $L_{2}$ (can be hidden/not declared) in the same direction in the space $R^{3}$, and in the line $L_{1}$ there is the points $p_{i}$, while in the line $L_{2}$ there is points $q_{i}$, where $i, j=1, \ldots, n$ so that on each of the lines the distance of adjacent points is same, and then one point $p_{i}$ is connected by curve $c_{i}$ just to a point $q_{j} . L_{1}$ and $L_{2}$ are called upper frame and bottom frame, respectively. $\sigma_{i}, i=1, \ldots, n$ are generators [7]. Computationally,

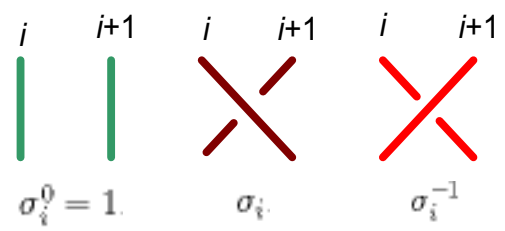

Fig. 1. Triple of woven patterns in braid

$$
\sigma_{i}^{1} \sigma_{i}^{-1}=\sigma_{i}^{0}=1
$$

Notation $\sigma_{i}{ }^{1}$ or simply written $\sigma_{i}$ is a woven geometric shape where the curve $c_{i}$ overpass $c_{i+1}$ exactly once on it, whereas $c_{j}$ for $j \neq i, i+1$ directly connects the points $p_{j}$ to $q_{j}$. Instead $\sigma_{i}{ }^{-1}$ is a woven where the curve $c_{i}$ underpass $c_{\mathrm{i}-1}$ exactly once on it, whereas $c_{j}, j \neq i-1, i$ is direct connect the points $p_{j}$ to $q_{j}$, see Fig. 2 [3]. The arrangement of points in a pair of frame lines can be expressed as a permutation form as follows

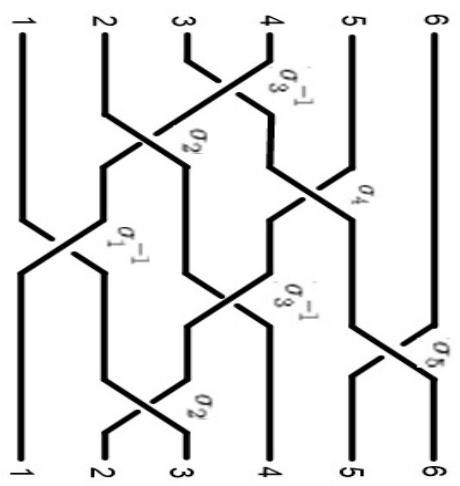

Fig. 2. A braid $A_{1}$ in $A_{6}$

$$
A=\left|\begin{array}{cccc}
1 & 2 & \ldots & N \\
c(1) & c(2) & \ldots & c(n)
\end{array}\right|
$$

$c$ is the curve that maps the points at $L_{1}$ to $L_{2}$. Number of generator $\sigma$ for representing $A$ is more than one woven (minimally is $\sigma^{0}$ ), and we denote it as $n_{\sigma}()$. For example, for $i=1,2, \ldots, 6$ we have one woven arrangement $A_{1}=$ $\sigma_{3}{ }^{-1} \sigma_{2} \sigma_{4} \sigma_{1}{ }^{-1} \sigma_{3}{ }^{-1} \sigma_{5} \sigma_{2}$ and $n_{\sigma}\left(A_{1}\right)=\left|\sigma_{3}{ }^{-1} \sigma_{2} \sigma_{4} \sigma_{1}{ }^{-1} \sigma_{3}{ }^{-1} \sigma_{5} \sigma_{2}\right|=7$, see Fig. 2, or based on Eq. (2) we have

$$
A_{1}=\left|\begin{array}{llllll}
1 & 2 & 3 & 4 & 5 & 6 \\
3 & 4 & 6 & 1 & 2 & 5
\end{array}\right|
$$

or $A=((1)(3)(6)(5)(2)(4))$.

A braid is a woven together with a deformation operation where no woven being of same height that

\footnotetext{
Corresponding author: mahyuddin@usu.ac.id
} 
during the deformation: (i) $L_{1}$ and $L_{2}$ remain parallel; (ii) There are no wedges between the curves; and (iii) The curve remains normal. The deformation can be expressed as a brushing, i.e. the projection of the curve $c_{i}$ moving from $p_{i}$ to $q_{j}$ has the distance from the line $L_{1}$ will always increase.

\subsection{Basic of braid group}

A set $A \neq \varnothing$ together with binary operation $\cdot$ denoted by $\langle A$,$\rangle is called a braid group if and only if: Binary$ operation between same ordered-braids can be expressed as a system [3], i.e. let $L_{1}, L_{2}, p_{i}, q_{j}, c_{i}$ and $L_{1}{ }^{\prime}, L_{2}{ }^{\prime}, p_{i}{ }^{\prime}, q_{i}{ }^{\prime}, c_{i}{ }^{\prime}$ respectively as frames, points, and curves of two braids $A$ in $A$ and $A^{\prime}$ in $A$, the braid $A \cdot A^{\prime}$ (or $A A^{\prime}$ ) can be obtained through the union of $L_{2}$ and $L_{1}{ }^{\prime}$ such that $q_{j}$ coincides with $p_{i}^{\prime}$ and $A A^{\prime}$ in $A$ is a new braid by removing $L_{2}=L_{1}{ }^{\prime}$; Thus, for every same ordered-braid, the associative law applicable, i.e. $\left(A_{1} A_{2}\right) A_{3}=A_{1}\left(A_{2} A_{3}\right), A_{1}, A_{2}, A_{3}$ in $\mathrm{A}$, Geometrically an identity braid, denoted by $A^{0}$, can be expressed as a woven arrangement by which each curve $c_{i}$ connects $p_{i}$ directly to $q_{j}$ and $i=j$, so that when the identity braid composed with any other same orderedbraid, the resulted braid will be equal to the origin braid. While, geometrically the inverse of $A$ can be guaranteed to exist through the results of reflection of $A$ to the line $L_{2}$ of $A$ so that $L_{2}$ becomes $L_{1}{ }^{\prime}$ of the braid $A^{-1}$, it constructed by the shadow of $L_{1}$ against $L_{2}$ whereby the curve $c_{i}^{\prime}$ will be the inverse of the curve $c_{i}$ and consequently the woven on $A$ where the curve $c_{i}$ overpass become the curve $c_{i}$ underpass $A^{-1}$, and their distance to $L_{2}$ and $L_{1}{ }^{\prime}$ be equal. That is $A A^{-1}=A^{0}=A^{-1} A$ and we conclude that $A^{0}=1$ where $A^{0}$ in $A$ and $A^{-1}$ in $A$ $[7,8]$.

The class of same ordered braids together with the operation is a group. This group is not determined by the length of the curve $c_{i}$ of each braid, but only by the woven structure of $\sigma$ in braids. We denoted $A_{n}$ as a group of braids order $n$. However, the set of permutations that represent all the braids is also a group called the $n$ ordered permutation group and denoted by $\mathscr{P}_{n}$ [9]. Depending on any arrangement of $\sigma_{j}$ can be added/ inserted to any braid provided that the $\sigma_{j}$ arrangement applies to $j<i$ while the other curve of $c_{i}$ connects the same point. Thus, the adding process of the $\sigma_{j}$ arrangement at the beginning or end of the braid is like performing a binary operation, whereas insertion is done by constructing a new line to cut and then adding new ones and combining other pieces based on appropriate binary operations.

For instance, let $A_{2}=\sigma_{1} \sigma_{4}{ }^{-1} \sigma_{2}$ for $i=1,2, \ldots, 6$ where $n_{\sigma}\left(A_{2}\right)=3$, then $A_{1} A_{2}=\left(\sigma_{3}^{-1} \sigma_{2} \sigma_{4} \sigma_{1}^{-1} \sigma_{3}{ }^{-1} \sigma_{5} \sigma_{2}\right)\left(\sigma_{1} \sigma_{4}{ }^{-1} \sigma_{2}\right)=$ $\sigma_{3}{ }^{-1} \sigma_{2} \sigma_{4} \sigma_{1}{ }^{-1} \sigma_{3}{ }^{-1} \sigma_{5} \sigma_{2} \sigma_{1} \sigma_{4}{ }^{-1} \sigma_{2}$ or by using Eq. (2) and Eq. (3) we obtain

$$
\begin{aligned}
A_{1} A_{2} & =((1)(3)(6)(5)(2)(4))(((1)(3)(2))((5)(4)))) \\
& =(((1)(2)(5))((3)(6)(4)))
\end{aligned}
$$

Thus $A_{1} A_{2} \neq A_{2} A_{1}$, but Eq. (4) is the direct proof of $\left(A_{1} A_{2}\right) A_{3}=A_{1}\left(A_{2} A_{3}\right)$. Naturally, suppose there is $A_{1}^{-1}=$ $\sigma_{2}{ }^{-1} \sigma_{5}{ }^{-1} \sigma_{3} \sigma_{1} \sigma_{4}{ }^{-1} \sigma_{2}{ }^{-1} \sigma_{3}$, then $A_{1} A_{1}^{-1}=\left(\sigma_{3}{ }^{-1} \sigma_{2} \sigma_{4} \sigma_{1}{ }^{-1} \sigma_{3}{ }^{-1} \sigma_{5} \sigma_{2}\right)$ $\left(\sigma_{2}^{-1} \sigma_{5}^{-1} \sigma_{3} \sigma_{1} \sigma_{4}^{-1} \sigma_{2}^{-1} \sigma_{3}\right)=\sigma_{3}^{-1} \sigma_{2} \sigma_{4} \sigma_{1}^{-1} \sigma_{3}{ }^{-1} \sigma_{5} \sigma_{2} \sigma_{2}{ }^{-1} \sigma_{5}{ }^{-1} \sigma_{3} \sigma_{1} \sigma_{4}{ }^{-}$ ${ }^{1} \sigma_{2}{ }^{-1} \sigma_{3}=\sigma_{3}{ }^{-1} \sigma_{2} \sigma_{4} \sigma_{1}{ }^{-1} \sigma_{3}{ }^{-1} \sigma_{5} \sigma_{5}{ }^{-1} \sigma_{3} \sigma_{1} \sigma_{4}{ }^{-1} \sigma_{2}{ }^{-1} \sigma_{3}=\ldots=1=A^{0}$, or by using Eq. (2) and Eq. (3) we get

$$
\begin{aligned}
A_{1} A_{2} & =((1)(3)(6)(5)(2)(4))((1)(4)(2)(5)(6)) \\
& =((1))
\end{aligned}
$$

Therefore, a set contains braids $A_{n}$ based on curves $c_{i}$, $i=1,2, \ldots, 6$ is a 6 -ordered braid group, we denoted it as $<A_{6},>$ or briefly $A_{6}[3]$.

\subsection{Definition and characteristics}

The braid group $A_{n}$ is group generated by $n-1$ generators $\sigma_{i}, i=1, \ldots, n-1$ with the relations [10].

$$
\sigma_{i} \sigma_{j}=\sigma_{j} \sigma_{i}, \text { for all } i, j=1,2, \ldots, n-1,|i-j| \geq 2
$$

and

$$
\sigma_{i} \sigma_{i+1} \sigma_{i}=\sigma_{i+1} \sigma_{i} \sigma_{i+1}, i=1,2, \ldots, n-2
$$

In algebra computation, let $\boldsymbol{a}=\sigma_{1} \sigma_{2} \ldots \sigma_{i-1} \sigma_{i} \sigma_{i+1} \ldots \sigma_{n-1}$, based on Eq. (6) and Eq. (7) we have $\boldsymbol{a} \sigma_{i}=\boldsymbol{a}_{\boldsymbol{i}+1} \boldsymbol{a}, i=$ $1, \ldots, n-2$, or $\sigma_{i+1}=\boldsymbol{a} \sigma_{i} \mathbf{a}^{-1}, \boldsymbol{a a}^{-1}=1$. After some of iterations we obtain $\sigma_{i}=\boldsymbol{a}_{i-1} \sigma^{-(i-1)}$.

Addition and insertion of structures such as Eq. (6) and Eq. (7) and their derivatives generally change the arrangement pattern in permutations that are an element of the $\mathscr{P}_{n}$ group. Therefore, there is a relation between two groups, $A_{n}$ and $\mathscr{P}_{n}$, as the classifications of elements in braid group.

\section{An Approach}

As has been expressed in its definition and characteristics, the woven arrangement that forms the braid gives a special meaning to each braid. Therefore, the braid group can be represented by two representations of structure and frame, the presence of the relation between two forms is a linkage that gives a special meaning. Thus, an approach is used to express the meaning of the structure and frames of the braid and relation between them. It is general expressed by group theory that there is a classification between braids that have the same meaning or in vary [11].

A mapping from the braid group $A_{n}$ to the $\mathscr{P}_{n}$ permutation group is to define the classification of braids into appropriate classes [9], i.e.

$$
h: A_{n} \rightarrow \mathscr{P}_{n}
$$

then the specific meaning is based on its structure through the composition of the generator that builds a braid. Disclosure of $\sigma$ with index composition will be different meaning in the presence of $\sigma^{-1}$ as opposed to. The ratio includes a comparison that assesses the braid structure. Likewise with the permutation groups, the same point ratio of positions on both frames connected by the same curve $c_{i}$ would differ in meanings of its 
presence compared to the presence of curve $c_{i}$ connecting the different points between the two frame lines [3]. Thus, it is a comparison about the structures or frames of braids and it gives the meaning of the existence of elements in braid group.

\section{The Structure of Braid}

Let $\sigma_{i}$ is one generator of braids whereby its permutation is

$$
P_{i}=\left|\begin{array}{cccc}
\ldots & i & j & \ldots \\
\ldots & j & i & \ldots
\end{array}\right|
$$

but for $\sigma_{i}^{2}$ the permutation is

$$
P_{0}=\left|\begin{array}{llll}
\ldots & i & j & \ldots \\
\ldots & i & j & \ldots
\end{array}\right|
$$

and we conclude that for $\sigma_{t}^{p}$ whereby $p$ is even or $p=$ $0,2,4, \ldots$ we have their permutations are like Eq. (10), while $p$ is odd or $p=1,3,5, \ldots$ we have their permutations are like Eq. (9). The composition of generator is not only based on one index but a sequential index as $\sigma_{i} \sigma_{i+1} \sigma_{i+2} \ldots$ $[8,3]$. Therefore, we have a lemma as follows.

\subsection{Relation}

Lemma 1. Let a braid with the composition of generators in $\left(\sigma_{i} \sigma_{i+1 \ldots} \sigma_{n-1}\right)^{p}$, if $p=n j, j=1,2, \ldots, k$ then their permutation is $P_{0}$.

Proof. Based on Eq. (9) and Eq. (10), $\sigma_{i} \sigma_{i+1}$ has a permutation $((i)(i+2)(i+1))$ and $\left(\sigma_{i} \sigma_{i+1}\right)^{3}$ has a permutation $((i))=((i)(i+2)(i+1))((i)(i+2)(i+1))((i)(i+2)$ $(i+1))$. Therefore, $n_{\sigma}()=3$ and $p=3$. For $\sigma_{i} \sigma_{i+1} \sigma_{i+2}$ has a permutation $((i)(i+3)(i+1)(i+2))$ we have $\left(\sigma_{i} \sigma_{i+1} \sigma_{i+2}\right)^{4}$ has a permutation $((i))$. Thus, $n_{\sigma}()=4$ or $p=4$. For $n_{\sigma}()=n$, then $p=n$ where $\left(\sigma_{i} \sigma_{i+1} \ldots \sigma_{n-1}\right)^{n}$ has $((\mathrm{i}))$. Therefore, because $((\mathrm{i}))((\mathrm{i}))=((\mathrm{i}))=P_{0},((\mathrm{i}))((\mathrm{i}))((\mathrm{i}))=((\mathrm{i}))=P_{0}$, $\ldots$, we simplify that for $p=n j, j=1,2, \ldots, k$, we have permutations of $\left(\sigma_{i} \sigma_{i+1} \ldots \sigma_{n-1}\right)^{n j}$ are $P_{0}$.

We call $\left(\sigma_{i} \sigma_{i+1} \ldots \sigma_{n-1}\right)^{n j}$ as the normal braids $[3,12]$.

Lemma 2. For each braid in a braid group, if there is one frame line on the braid then the line cuts the braid be two braid in a braid group.

Proof. Let $A$ in $A$, the line cut the braid right on the $\sigma_{i}^{0}$ for $i=1,2, \ldots, n$ so that there two braids: $A_{1}, A_{2}$ in $A$ or $A^{0}, A$ in $A$, we call them as the resultant braids, and be in force $A$ $=A_{1} A_{2}, A=A^{0} A$, or $A=A A^{0}$. Because on a new frame line there are corresponding points then each resultant braid has a permutation.

Proposition 1. For each braid can be inserted other braid.

Proof. To insert any braid into another braid is by first considering the curves $c_{i}$ connecting two frame lines. If both braids are in the same order, then the insertion can be done. If the number of curves $c_{i}$ of the inserted braid is smaller than the number of curves $c_{j}$ of braid accept the insertion, then the number of curves $c_{i}$ is adjusted to the number of curves $c_{j}$ by adding curves in the appropriate part. Also it applies vice versa. Therefore, based on Lemma 2, insertion can be done by separating one braid into two braids, and then carrying out binary operations for the three braids by order first braid, the inserted braid, and the last braid.

Theorem 1. For the normal braids, the insertion of the normal braids to any other braid does not alter its permutation.

Proof. First, based on Lemma 1 the normal braids has $P_{0}$, based on Proposition 1 the normal braids can be inserted to any braids, and based on Lemma 2 we have a new braid with applying the binary operation on three braids. In other words, $A=A_{1} A_{2}$ in $A_{n}$, we have $A=$ $A_{1}\left(A^{0}\right) A_{2}$ whereby the permutation of $A$ do not change. Second, let braid $A$ has a permutation $((i)(j))$, based on Proposition 1 we have two permutations $((\ldots)(i)(k)(j)$ $(\ldots))$ and $((\ldots)(k)(j)(\ldots))$ for example and we have $((i)((j))=((\ldots)(i)(k)(j)(\ldots))((i))((\ldots)(k)(j)(\ldots))$, whereby $((i))$ is presentation of the normal braids.

Corollary 1. The insertion of un-normal braids to any other braid alters its permutation.

Proof. This is as consequence of Theorem 1 to Proposition 1.

\subsection{Defining characteristics}

Through Lemma 1, Lemma 2, Proposition 1 and Theorem 1 has been shown that there is a function $h$ from $A_{n}$ into $\mathscr{P}_{n}$, that is

$$
h(N: 1): A_{n} \rightarrow \mathscr{P}_{n}
$$

which classifies each element of $A_{n}$ into the same class based on elements of $\mathscr{O}_{n}$.

Computationally, it can be declared some scales of calculation in braid group $A_{n}$, i.e.

[S1] A number of generators allowed in the braid group $A_{n}$, we denoted it as $n_{n-1}()$, for example the braid group $A_{6}$ has $n_{6}=5$, or generally the braid group $A_{n}$ based on definition of braid group having $n_{n}()=n-1$.

[S2] A number of generators that appears in a braid, this is expressed as the cardinality we denoted it as $n_{c}()=\|$.$\| .$ For example $n_{c}\left(A_{1}\right)=\left\|\sigma_{3}{ }^{-1} \sigma_{2} \sigma_{4} \sigma_{1}{ }^{-1} \sigma_{3}{ }^{-1} \sigma_{5} \sigma_{2}\right\|=5$ because only $\sigma_{1}, \sigma_{2}, \sigma_{3}, \sigma_{4}$ and $\sigma_{5}$ that appear in a braid $A_{1}=\sigma_{3}{ }^{-}$ ${ }^{1} \sigma_{2} \sigma_{4} \sigma_{1}{ }^{-1} \sigma_{3}{ }^{-1} \sigma_{5} \sigma_{2}$, while $A_{1} A_{2}=\sigma_{3}{ }^{-1} \sigma_{2} \sigma_{4} \sigma_{1}{ }^{-1} \sigma_{3}{ }^{-1} \sigma_{5} \sigma_{2} \sigma_{1} \sigma_{4}{ }^{-1} \sigma_{2}$ also has $n_{c}\left(A_{1} A_{2}\right)=\left\|\sigma_{3}^{-1} \sigma_{2} \sigma_{4} \sigma_{1}{ }^{-1} \sigma_{3}{ }^{-1} \sigma_{5} \sigma_{2} \sigma_{1} \sigma_{4}{ }^{-1} \sigma_{2}\right\|=5$. Minimum of $n_{c}\left(\right.$ ) is 0 (zero) because $\sigma_{i}^{0}=1$. Therefore, 0 $\leq n_{c} \leq n-1$.

[S3] A size of woven arrangement in a braid, we redefined it as $n_{\sigma}()=\mid$.|. For example, $n_{\sigma}\left(A_{1}\right)=\mid \sigma_{3}{ }^{-1} \sigma_{2} \sigma_{4} \sigma_{1}{ }^{-}$ ${ }^{1} \sigma_{3}{ }^{-1} \sigma_{5} \sigma_{2} \mid=7$, while $n_{\sigma}\left(A_{1} A_{2}\right)=\mid \sigma_{3}{ }^{-1} \sigma_{2} \sigma_{4} \sigma_{1}{ }^{-1} \sigma_{3}{ }^{-1} \sigma_{5} \sigma_{2} \sigma_{1} \sigma_{4}{ }^{-1}$ $\sigma_{2} \mid=10$. Based on $n_{c}()$ we have also minimum of $n_{\sigma}()$ is 0 (zero), but maximum of $\mathrm{n}_{\sigma}()$ cannot be determined (it is uncertainty). Thus, $0 \leq n_{\sigma}() \leq \ldots$.

To generate the potential about the structure of the braids, the ratio between three measurements: S1, S2 and $\mathrm{S} 3$ give the different meaning. That ratio between $\mathrm{S} 1$ and $\mathrm{S} 2$,

$$
r_{c}()=n_{c}() / n_{n-1}()
$$


where $0 \leq r_{c} \leq 1$. It gives special meaning as a probability of a braid about structure in a braid group. In other case, we can generate the potential strength of braid in a braid group by ratio between $\mathrm{S} 1, \mathrm{~S} 2$ and $\mathrm{S} 3$,

$$
r_{\sigma}()=n_{\sigma}() / n_{c}()
$$

where $0 \leq r_{c} \leq \ldots$, and if $r_{\sigma}$ divided by $n$ - 1 we have average of a braid structure or $r_{a v}()$. Let $A_{1}, A_{2}$ in $A_{6}$, we obtain $n_{n}\left(A_{6}\right)=5$, and then $r_{c}\left(A_{1}\right)=5 / 5=1.0000, r_{c}\left(A_{2}\right)$ $=3 / 5=0.6000$, but $r_{c}\left(A_{1} A_{2}\right)=5 / 5=1.0000$ also. Therefore, to get another meaning of structure in a braid, we use Eq. (13) and their results divided by $n-1$. That is $r_{a v}\left(A_{1}\right)=r_{\sigma}\left(A_{1}\right) / n_{n}\left(A_{6}\right)=\left(n_{\sigma}\left(A_{1}\right) / n_{c}\left(A_{1}\right)\right) / n_{n}\left(A_{6}\right)=(7 / 5) / 5=$ 0.2800. Similarly, $r_{a v}\left(A_{2}\right)=0.2000$ and $r_{a v}\left(A_{1} A_{2}\right)=$ 0.4000 . Thus, although $r_{c}\left(A_{1}\right)=r_{c}\left(A_{1} A_{2}\right)$, but $r_{a v}\left(A_{1}\right) \neq$ $r_{a v}\left(A_{1} A_{2}\right)$.

In permutation, it can declare some scales of calculation in permutation group $\mathscr{P}_{n}$, i.e.

[S4] Number of points in the frame line, we denoted it as $n_{n}()=n$. For example, $n_{n}(\mathscr{O})=6$ for a group braid $A_{6}$.

[S5] Number of points in same position on two frame lines, we denoted it as $n_{p}()$. For example, for a braid $A_{1}$ we have $n_{p}\left(A_{1}\right)=6$.

[S6] Number of points in different position on two frame lines, we denoted it as $n_{q}()$. For example, for a braid $A_{1}$ we obtain $n_{q}\left(A_{1}\right)=0$.

If probability of same position of points in two frame lines is

$$
r_{p}()=n_{p}() / n_{n}(), 0 \leq r_{p}() \leq 1,
$$

and probability of different position of points in two frame lines is

$$
r_{q}()=n_{q}() / n_{n}(), 0 \leq r_{q}() \leq 1,
$$

then $r_{p}()=1-r_{q}()$. For example, $r_{p}\left(A_{1}\right)=6 / 6=1$ and $r_{q}\left(A_{1}\right)=0 / 6=0$. Thus Eq. (12), (13), 14), and (15) redefine $h$ on Eq. (11) for a braid group and a permutation group.

\section{The conclusion}

By involving the definition of braid groups, several characteristics are presented to reveal the meaning of each braid. A function $h$ has given relation between braid group and permutation group, it has shown the characteristics expressed through Eq. (12), (13), (14), and (15) give different meanings for each braid structure. This also includes the braids classification.

\section{References}

1. E. Artin Hamb. Abs. 4 (1925).

2. D. L. Goncalves Indagationes Mathematicae 29, 1 (2018).

3. M. K. M. Nasution Jurusan Matematika, FMIPA USU (1992).
4. W. B. Bauer, F. H. C. Crick, J. H. White Scientific American 243 (1980).

5. M. K. M. Nasution Jurnal Matematika dan Terapannya 1, 2 (2000).

6. D-m Tong, S-d Yang, Z-q Ma Communications in Theoretical Physics 26, 4 (2018).

7. E. Artin Annals of Mathematics 48 (1947).

8. E. Artin American Scientist 38 (1950).

9. N. V. Ivanov Mathematics of the USSR-Sbornik 71, 2 (1992).

10. R. Fox, L. Neuwirth Mathematica Scandinavica 10 (1962).

11. T. E. Brendle, D. Margalit Journal fur die reine und angewandte Mathematik 735 (2018).

12. M. K. M. Nasution, A. S. Harahap, S. Nasution, E. Herawati IOP Conference Series: Materials Science and Engineering 300, 1 (2018). 